\title{
Effect of 1-Methylcyclopropene and Polyhexamethylene Guanidine on Postharvest Quality of Banana (Musa paradisiaca) cv. Yelakki
}

\author{
S.B. Gouri ${ }^{1 *}$, H.C. Krishna ${ }^{1}$, T.H. Shankarappa ${ }^{1,2}$, K.N. Sreenivas ${ }^{1,2}$, \\ G.K. Sadananda ${ }^{1}$, D.V. Sudhakar Rao ${ }^{3}$ and Shamina Azeez ${ }^{3}$ \\ ${ }^{1}$ Department of Postharvest Technology, College of Horticulture, University of Horticultural \\ Sciences Campus, GKVK, Bengaluru, 560 065, India \\ ${ }^{2}$ College of Horticulture, Tamaka, Kolar 563 103, India \\ ${ }^{3}$ Department of Postharvest Technology, Indian Institute of Horticultural Research, \\ Bengaluru, 560 089, India \\ *Corresponding author
}

\section{A B S T R A C T}

\begin{tabular}{|l|}
\hline Ke y w o r d s \\
Banana, Colour \\
change, Decay loss, \\
1-MCP, PHMG, \\
Respiration rate, \\
Shelf life. \\
\hline Article Info \\
\hline $\begin{array}{l}\text { Accepted: } \\
\text { 24 February } 2017 \\
\text { Available Online: } \\
\text { 10 March } 2017\end{array}$ \\
\hline
\end{tabular}

A study was undertaken to manage two factors that are responsible for reduced market value in banana cv. Yelakki namely ethylene biosynthesis and microbial deterioration. Freshly harvested stage 2 banana fruits treated with chemicals, 1-methylcyclopropene (1$\mathrm{MCP}$ ) and poly hexa methylene guanidine (PHMG) at different concentrations and stored in corrugated fiber board boxes at ambient condition for 15 days. The fruits treated with combination of 1-MCP (20 ppm) and PHMG (2.0\%) showed lower fruit respiration rate (0.02-0.15mg CO $\mathrm{kg}^{-1} \mathrm{~h}^{-1}$ ), lower PLW (3.89-18.83\%) and low decay loss (16.54\%). This treatment was also found to have components such as chlorophyll content, firmness, ascorbic acid, total soluble solids, total sugars and pectin, mineral contents viz. calcium, magnesium and potassium on higher side at the end of 15 days storage. Regression studies of the experiment showed a positive and significant association with ripening rate $(0.43)$, colour change (0.56), PLW (0.85), moisture (0.16), TSS (0.49), total sugars (0.62), pectin (0.43), calcium (0.35), potassium (0.29) and decay loss (0.64) and it was negative for firmness (-0.29) and ascorbic acid (-0.15). The treated fruits showed significantly higher shelf life of 15 days however, the scores of sensory analysis viz. colour, taste, texture, flavor and overall acceptability were poor.

\section{Introduction}

Banana (Musa paradisiaca) is one of the most important tropical fruit crops of the family Musaceae, native to tropical regions of SouthEast Asia and cultivated widely in subtropical regions. The cultivar Yelakki banana is a geographical indicator of Mysore, Karnataka, India (Usha Rani and Kishor Kumar, 2013). Banana, being a climacteric fruit it is harvested at different maturity levels based on purpose and distance of transportation. It has two systems of ethylene production.

System 1, a low basal rate of ethylene production occurs during maturation and system 2, a characteristic autocatalytic climacteric rise in ethylene production (McMurchie et al., 1972) after maturation. 
Commercially, bananas are induced to ripen with ethylene which reduces the marketing life from 3 to 5 days depending on ethylene treatment conditions and holding temperature after treatment. Generally fruit ripening is characterized by loss of green colour, softening of pulp, accelerated metabolic process followed by shriveling and rotting (Chun, 2010). The presence of ethylene in the atmosphere has been a major concern for unripe climacteric fruits during postharvest handling, as it is known to accelerate ripening, senescence, abscission and physiological disorders. The action of ethylene must be avoided during storage and transportation to limit the ethylene biosynthesis and fast removal of ethylene emitted from the surrounding atmosphere of produce. A simple physical method to prevent ethylene accumulation is to ensure good air circulation inside the storage room and ventilation (Wu, 2010). Use of ethylene absorbents such as potassium permanganate on vermiculite in packages are in vogue for oxidizing ethylene release from fresh produce and enhancing the shelf life of fruits (Pereira Silva et al., 2009). A new innovative tool, 1methylcyclopropene (1-MCP) an ethylene action inhibitor, has been added to the above list of options for extending the shelf life and quality of fresh horticultural produce. At standard temperature and pressure, 1-MCP is a gas with a molecular formula of $\mathrm{C}_{4} \mathrm{H}_{6}$. It is thought to occupy ethylene receptors such that ethylene cannot bind and elicit action (Pereira Silva et al., 2009). The affinity of 1MCP for the receptor is approximately 10 times greater than that of ethylene and compared with ethylene, 1-MCP is active at much lower concentrations (Sisler and Serek, 1997).

Spoilage is another problem in banana fruits caused by microorganisms mainly anthracnose, cigar end rot and crown rot caused by Colletotrichum, Verticillium and
Acremonium, respectively both in field and after harvest. Postharvest diseases can cause serious losses of fruits both in terms of quantity and quality (Nelson, 2008). Poly hexa methylene guanidine (PHMG) is one efficient, multipurpose cationic polymer used as bactericide, fungicide, disinfectant, preservative and sanitizer. Its action is very fast and its single molecular nature works as a great disinfectant, at least 20 times and up to 1000 times more effective than silver and $\mathrm{H}_{2} \mathrm{O}_{2}$ respectively. It is a safe and environmentally friendly type of disinfectant, decontaminates the ready to eat fruits and vegetables, extends the shelf life and protects the raw and post harvest fruits and vegetables. PHMG has zero toxicity and no irritation to human's skin.

The postharvest life of banana is limited by physiological deterioration, which leads to decay via disease development and since the fruits contain a wide range of organic substrates, high water activity, and thus are good substrates for microbial spoilage. There is a need to monitor and manage the fruit quality and disease severity during postharvest life of banana, hence the effect of 1-methylcyclopropene and poly hexa methylene guanidine to control ethylene biosynthesis and microbial deterioration on postharvest quality of banana (musa paradisiaca) cv. Yelakki is studied.

\section{Materials and Methods}

Banana cv. 'Yelakki' was harvested at mature green (second stage), from the farm practicing good horticultural practices nearby experimental site. The harvesting was done manually in the cool hours; harvesting maturity was decided based on criteria such as change in fruit skin colour from dark green to light green, reduced fruit detachment force and disappearance of angularity on the fingers. Fruits were subjected to sorting and 
grading operation on arrival to the laboratory to maintain homogeneity in the experiment. The fruits of banana cv. Yelakki were divided into five lots and each lot (treatment) contained two $\mathrm{kg}$ of fruits.

There were treatments; $\mathrm{T}_{1}$ - Control, $\mathrm{T}_{2}$ PHMG @1.50\%+1-MCP @ 15ppm, T $3^{-}$ PHMG@1.50\%+1-MCP @ 20ppm, $\mathrm{T}_{4}$ PHMG@2.0\%+1-MCP @15ppm and $\mathrm{T}_{5}$ PHMG@2.0\%+1-MCP @ 20ppm and were replicated four times.

The required quantity and concentration of PHMG solution was prepared freshly for application on fruits, 1.50 and 2.0 per cent of PHMG solution was prepared using distilled water, fruits were dipped in the solution for 5 minutes and air dried and then subjected for 1-MCP fumigation.

The tablet form of 1-MCP was powdered and 15 and $20 \mathrm{mg}$ of 1-MCP dissolved in $1000 \mathrm{ml}$ of distilled water to obtain 15 and 20 ppm solution respectively. $20 \mathrm{ml}$ of each concentration was taken in a $25 \mathrm{ml}$ beaker and placed in air tight zip lock polyethylene covers along with banana fruits for fumigation immediately after preparation at ambient condition. The fruits were removed from fumigation chamber after 6 hours of exposure. Banana fruits treated with both chemical along with control were stored in corrugated fibre board boxes at ambient temperature condition $\left(25\right.$ to $\left.31^{\circ} \mathrm{C}\right)$ and relative humidity (51 to $70 \%$ ).

The parameters like firmness, TSS, titratable acidity, reducing sugar, total sugar, ascorbic acid, pectin content, mineral content respiration rate and decay loss were estimated by using standard procedures (AOAC, 1980). A visual colour score was given to each stage of fruit ripening and the co-efficient of ripening was calculated using the following formula.
Coefficient of ripening $=$

$\sum$ (No. of fruits at a particular ripening stage $\mathrm{x}$ its score

\section{Total number of fruits}

Ripeness test was also done by using NIR (Near infrared) instrument (DA meter, Make: Bologna, Italy) based on chlorophyll degradation and same is correlated with the TSS, firmness, acidity, ascorbic acid, sugars, pectin and minerals.

Respiration rate was measured using the static head space technique. Five fruits from each replication were selected at random and enclosed in a hermetically sealed container $(3000 \mathrm{~mL})$ fitted with a silicon rubber septum for 3 hours. The concentrations of $\mathrm{O}_{2}$ and $\mathrm{CO}_{2}$ were recorded in the head space of the container by piercing the probe of an auto oxygen / carbon dioxide analyzer (Make: Quantek, Model: 902D Dual track) into the container through the septa fixed on the lid of container and direct reading was noted down from the instrument screen. The $\mathrm{CO}_{2}$ evolution was expressed in $\mathrm{mg} \mathrm{kg}^{-1} \mathrm{~h}^{-1}$.

Fruits from each treatment were taken to record the physiological loss in weight. The weight of the fruits was recorded using electronic weighing balance (Model: Essae, DS-852, Teraoaka Ltd.) before storage. Thereafter, the weights were recorded regularly during storage.

The firmness of the fruit, at equatorial region, was measured as the force required for puncturing the fruits using a fruit firmness tester (Model FT110, Make: Tarnai, Italy). A probe of $8 \mathrm{~mm}$ diameter was used, with $13 \mathrm{~kg}$ capacity. The firmness was recorded and expressed as $\left(\mathrm{kg} \mathrm{cm}^{2-1}\right)$. The moisture of banana fruit was measured at regular intervals. Five grams of fruit pulp sample was taken and cut in to small pieces and placed in 
Sartorius electronic moisture analyzer (Model: MA 35) and the direct reading was noted down from the instrument screen and expressed in per cent.

Banana fruits were finally assessed for their sensory features such as color, taste, flavor, texture / body and absence of defects before and after storage period as initial and final observation by a panel of judges using composite scoring test (Barrett, 2010). All the experiments were laid out in simple completely randomized design (CRD), were analyzed by using SPSS 16.0 software (Sunder Raj et al., 1972) and also subjected for regression analysis.

\section{Results and Discussion}

\section{Effect of 1-MCP and PHMG on fruit ripening rate}

The ripening rate of banana fruits is expressed as co-efficient of ripening with a score of 1 to 7 , where 1 indicates unripe stage and 7 indicates over ripe stage. As shown in Fig.1, the ripening rate of the banana fruits during storage increased from the harvest till the end of their storage life, irrespective of the pretreatments given. However, as depicted in Table 1, there was a significant delay in the ripening rate of $1-\mathrm{MCP}$ and $\mathrm{PHMG}$ treated fruits at all the treatments except control.

The delayed ripening of banana when treated with 1-MCP is scientifically documented by the fact that 1-MCP is strong inhibitor of ethylene. Upon harvesting of banana fruits, they continue to produce ethylene through methionine via intermediates S-adenosylmethionine (SAM) and 1-aminocyclopropane1-carboxylic acid (ACC). ACC synthase (ACS) convert SAM to ACC and ACC Oxidase (ACO) convert ACC to ethylene, where ready for its ripening action. At this stage 1-MCP is known to bind ethylene receptors in banana fruit, giving no chances for ethylene action, transformed in to low respiration rate and hence longer shelf life. Over the time, the fruits start to synthesize new ethylene receptors thus returning the ethylene sensitivity. Similar results of reduced ripening rate by $1-\mathrm{MCP}$ were confirmed by (McMurchie et al., 1972; Saltveit, 1999; Yueming et al., 1999; Kader and Saltveit, 2003; Kays and Paull, 2004; Barry and Giovannoni, 2007; Wills et al., 2007; Lin et al., 2009).

\section{Effect of 1-MCP and PHMG on respiration rate}

The respiration rate of banana fruits treated with 1-MCP and PHMG in combination had shown a significantly lower respiration rate than control fruits throughout their storage life. As evident from the Table 2, the respiration rate had increased rapidly from initial $1^{\text {st }}$ day to $7^{\text {th }}$ day of storage. However, 1-MCP and PHMG treatments had shown a significantly lower respiration rate on the $7^{\text {th }}$ day. The combination effect of 1-MCP and PHMG showed a gradual increase in respiration rate up to $15^{\text {th }}$ day of storage.

One of the very basic principles of postharvest management of banana fruit is to reduce respiration rate there by prolonging the shelf life. This principle is addressed in this study by use of 1-MCP on fruits.

Bananas treated with 1-MCP had suppressed the respiration rate which indicates the role of ethylene in triggering the respiration rate of banana fruits. In the present study, reduced respiration rate was found in 1-MCP treated fruits as presented in the Fig.1. The suppression of respiratory rates of banana fruits in response to 1-MCP treatment has been reported previously (Clara et al., 2002; Eduardo et al., 2006). 
Table.1 Effect of 1-methylcyclopropene and polyhexamethylene guanidine on ripening rate of banana fruits during storage

\begin{tabular}{|c|c|c|c|c|c|c|c|c|c|c|c|c|c|c|c|c|c|c|c|c|}
\hline Treatments & D1 & D2 & D3 & D4 & D5 & D6 & D7 & D8 & D9 & D10 & D11 & D12 & D13 & D14 & D15 & D16 & D17 & D18 & D19 & D20 \\
\hline T1 & 1.32 & 1.32 & 3.55 & 4.69 & 5.17 & 5.49 & 7.00 & --- & --- & --- & --- & --- & --- & --- & --- & --- & --- & --- & --- & --- \\
\hline T2 & 1.29 & 1.29 & 2.55 & 2.82 & 3.47 & 3.47 & 3.47 & 3.47 & 3.94 & 3.94 & 5.45 & -- & --- & --- & --- & --- & -- & --- & --- & -- \\
\hline T3 & 1.23 & 1.23 & 2.30 & 3.94 & 3.82 & 3.82 & 3.82 & 3.82 & 3.94 & 3.94 & 5.54 & --- & --- & --- & --- & --- & --- & --- & --- & --- \\
\hline T4 & 1.33 & 1.33 & 2.32 & 5.76 & 5.76 & 5.76 & 5.76 & 5.76 & 5.78 & 5.78 & 5.76 & --- & --- & --- & --- & --- & --- & --- & --- & --- \\
\hline T5 & 1.20 & 1.20 & 2.78 & 4.35 & 4.35 & 4.35 & 4.35 & 4.35 & 4.35 & 4.35 & 4.34 & --- & --- & --- & --- & --- & --- & --- & --- & --- \\
\hline T6 & 1.29 & 1.29 & 1.29 & 1.52 & 1.52 & 1.52 & 1.52 & 1.52 & 2.41 & 2.41 & 2.41 & 3.02 & 3.02 & 3.02 & 3.35 & 3.65 & 4.12 & 4.12 & 4.35 & 5.65 \\
\hline T7 & 1.16 & 1.16 & 1.16 & 1.23 & 1.23 & 1.23 & 1.23 & 1.23 & 2.57 & 2.57 & 2.57 & 2.70 & 2.70 & 2.94 & 3.84 & 3.87 & 4.66 & 4.66 & 4.86 & 5.17 \\
\hline T8 & 1.12 & 1.12 & 1.12 & 1.16 & 1.16 & 1.16 & 1.16 & 1.16 & 2.66 & 2.66 & 2.66 & 2.90 & 2.90 & 2.90 & 3.08 & 3.54 & 4.51 & 4.51 & 4.35 & 5.43 \\
\hline T9 & 1.18 & 1.18 & 1.18 & 1.17 & 1.17 & 1.17 & 1.17 & 1.17 & 2.56 & 2.56 & 2.56 & 2.87 & 2.87 & 2.87 & 3.04 & 3.40 & 4.11 & 4.11 & 4.39 & 5.35 \\
\hline S. Em \pm & 0.003 & 0.003 & 0.005 & 0.009 & 0.048 & 0.048 & 0.049 & 0.048 & 0.007 & 0.007 & 0.008 & 0.010 & 0.010 & 0.010 & 0.014 & 0.006 & 0.005 & 0.005 & 0.006 & 0.006 \\
\hline CD@ $@ 5 \%$ & 0.010 & 0.010 & 0.015 & 0.028 & 0.139 & 0.139 & 0.144 & 0.139 & 0.021 & 0.021 & 0.024 & 0.029 & 0.029 & 0.029 & 0.040 & 0.018 & 0.016 & 0.016 & 0.016 & 0.019 \\
\hline
\end{tabular}

Treatments: T1: control, T2: PHMG @ 0.5\%, T 3 PHMG @ 1\%, T 4 PHMG @ 1.5\%, T 5 PHMG @ 2.0\%, T61-MCP @ 5ppm, T71-MCP @ 10ppm, T81-MCP @ $15 \mathrm{ppm}, \mathrm{T}_{9} 1-\mathrm{MCP} @ 20 \mathrm{ppm}$

Table.2 Effect of 1-methylcyclopropene and polyhexamethylene guanidine on respiration rate (mg CO2 kg-1 h-1) of banana fruits during storage

\begin{tabular}{|c|c|c|c|c|c|c|c|c|c|c|c|c|c|c|c|c|c|c|c|}
\hline Treatments & D2 & D3 & D4 & D5 & D6 & D7 & D8 & D9 & D10 & D11 & D12 & D13 & D14 & D15 & D16 & D17 & D18 & D19 & D20 \\
\hline T1 & 0.09 & 0.11 & 0.13 & 0.14 & 0.24 & 0.28 & --- & -- & -- & -- & -- & --- & --- & --- & --- & --- & --- & -- & --- \\
\hline $\mathbf{T} 2$ & 0.08 & 0.10 & 0.11 & 0.12 & 0.15 & 0.21 & 0.23 & 0.24 & 0.24 & 0.26 & --- & --- & --- & --- & --- & --- & --- & --- & --- \\
\hline T3 & 0.08 & 0.09 & 0.10 & 0.11 & 0.15 & 0.21 & 0.23 & 0.24 & 0.25 & 0.26 & --- & --- & --- & --- & --- & --- & --- & --- & --- \\
\hline T4 & 0.07 & 0.09 & 0.09 & 0.11 & 0.14 & 0.22 & 0.22 & 0.23 & 0.24 & 0.27 & --- & --- & --- & --- & --- & --- & --- & --- & --- \\
\hline T5 & 0.06 & 0.09 & 0.10 & 0.11 & 0.14 & 0.20 & 0.21 & 0.22 & 0.23 & 0.25 & -- & --- & --- & --- & --- & --- & -- & --- & --- \\
\hline T6 & 0.01 & 0.02 & 0.02 & 0.02 & 0.02 & 0.03 & 0.03 & 0.04 & 0.06 & 0.07 & 0.07 & 0.08 & 0.07 & 0.10 & 0.09 & 0.15 & 0.19 & 0.22 & 0.26 \\
\hline $\mathbf{T} 7$ & 0.01 & 0.02 & 0.02 & 0.02 & 0.03 & 0.03 & 0.03 & 0.04 & 0.04 & 0.05 & 0.07 & 0.08 & 0.08 & 0.08 & 0.10 & 0.12 & 0.17 & 0.20 & 0.26 \\
\hline T8 & 0.01 & 0.01 & 0.01 & 0.01 & 0.02 & 0.02 & 0.02 & 0.03 & 0.03 & 0.04 & 0.05 & 0.06 & 0.07 & 0.07 & 0.09 & 0.11 & 0.15 & 0.17 & 0.24 \\
\hline T9 & 0.01 & 0.01 & 0.01 & 0.01 & 0.01 & 0.02 & 0.02 & 0.04 & 0.04 & 0.04 & 0.05 & 0.05 & 0.06 & 0.06 & 0.08 & 0.09 & 0.13 & 0.15 & 0.22 \\
\hline S Em \pm & 0.000 & 0.001 & 0.001 & 0.001 & 0.001 & 0.003 & 0.002 & 0.003 & 0.003 & 0.003 & 0.000 & 0.000 & 0.000 & 0.000 & 0.000 & 0.002 & 0.000 & 0.000 & 0.002 \\
\hline CD 5\% & 0.001 & 0.000 & 0.003 & 0.004 & 0.004 & 0.009 & 0.005 & 0.008 & 0.008 & 0.007 & 0.001 & 0.001 & 0.001 & 0.001 & 0.001 & 0.005 & 0.001 & 0.001 & 0.006 \\
\hline
\end{tabular}

Initial respiration: $0.012\left(\mathrm{mg} \mathrm{CO}_{2} \mathrm{~kg}^{-1} \mathrm{~h}^{-1}\right.$ 
Table.3 Effect of 1-methylcyclopropene and polyhexamethylene guanidine on cumulative physiological loss in weight (\%) of banana fruits during storage

\begin{tabular}{|c|c|c|c|c|c|c|c|c|c|c|c|c|c|c|c|c|c|c|c|}
\hline Treatments & D2 & D3 & D4 & D5 & D6 & D7 & D8 & D9 & D10 & D11 & D12 & D13 & D14 & D15 & D16 & D17 & D18 & D19 & D20 \\
\hline T1 & 0.89 & 2.45 & 3.05 & 5.94 & 7.72 & 12.18 & -- & -- & -- & -- & -- & --- & --- & -- & -- & -- & -- & -- & -- \\
\hline $\mathbf{T} 2$ & 0.90 & 2.78 & 3.27 & 5.27 & 7.52 & 9.59 & 12.05 & 12.88 & 14.90 & 16.58 & --- & --- & --- & --- & --- & --- & --- & --- & --- \\
\hline T3 & 0.86 & 2.16 & 2.90 & 4.81 & 6.54 & 8.43 & 10.04 & 11.22 & 15.57 & 17.09 & --- & --- & --- & --- & --- & --- & --- & --- & --- \\
\hline $\mathbf{T 4}$ & 0.88 & 2.14 & 3.30 & 5.34 & 6.31 & 7.83 & 11.02 & 11.87 & 12.53 & 14.99 & --- & --- & --- & --- & --- & --- & --- & --- & --- \\
\hline T5 & 0.78 & 1.91 & 3.16 & 5.19 & 7.33 & 6.38 & 9.84 & 13.04 & 13.37 & 14.77 & --- & --- & --- & --- & --- & --- & --- & --- & --- \\
\hline T6 & 0.59 & 1.44 & 1.96 & 3.43 & 4.19 & 5.14 & 5.94 & 6.20 & 6.85 & 7.73 & 8.71 & 9.60 & 10.32 & 11.62 & 12.60 & 13.62 & 16.79 & 18.28 & 19.41 \\
\hline T7 & 0.69 & 1.59 & 1.96 & 3.62 & 4.57 & 5.33 & 6.27 & 6.71 & 8.24 & 9.32 & 10.53 & 11.67 & 12.67 & 14.59 & 16.47 & 17.71 & 19.27 & 20.44 & 22.04 \\
\hline T8 & 0.60 & 1.48 & 1.43 & 2.98 & 3.57 & 4.40 & 4.93 & 5.61 & 6.51 & 7.00 & 8.25 & 8.96 & 9.85 & 9.80 & 10.75 & 11.81 & 13.13 & 16.14 & 18.35 \\
\hline T9 & 0.65 & 1.35 & 1.58 & 2.79 & 3.17 & 4.38 & 4.67 & 5.24 & 6.56 & 7.40 & 8.15 & 8.64 & 9.51 & 10.62 & 11.70 & 12.31 & 12.70 & 17.41 & 18.50 \\
\hline S Em \pm & 0.02 & 0.10 & 0.08 & 0.15 & 0.11 & 0.14 & 0.16 & 0.17 & 0.13 & 0.18 & 0.11 & 0.11 & 0.14 & 0.05 & 0.04 & 0.06 & 0.14 & 0.07 & 0.06 \\
\hline CD $5 \%$ & 0.06 & 0.30 & 0.24 & 0.45 & 0.31 & 0.40 & 0.46 & 0.49 & 0.37 & 0.52 & 0.31 & 0.32 & 0.42 & 0.13 & 0.10 & 0.17 & 0.41 & 0.22 & 0.18 \\
\hline
\end{tabular}

Table.4 Effect of 1-methylcyclopropene and polyhexamethylene guanidine on firmness $(\mathrm{kg} \mathrm{cm} 2-1)$ of banana fruits during storage

\begin{tabular}{|c|c|c|c|c|c|c|c|c|c|c|c|c|c|c|c|c|c|c|c|}
\hline Treatments & D2 & D3 & D4 & D5 & D6 & D7 & D8 & D9 & D10 & D11 & D12 & D13 & D14 & D15 & D16 & D17 & D18 & D19 & D20 \\
\hline T1 & 8.33 & 7.11 & 6.48 & 3.89 & 2.74 & 1.71 & --- & --- & --- & --- & --- & --- & --- & --- & --- & --- & --- & --- & --- \\
\hline $\mathbf{T} 2$ & 8.69 & 7.68 & 6.68 & 5.30 & 4.05 & 3.80 & 3.50 & 2.58 & 2.41 & 1.93 & --- & --- & --- & --- & --- & --- & --- & --- & --- \\
\hline T3 & 8.56 & 8.25 & 7.95 & 7.50 & 6.80 & 3.86 & 3.31 & 2.73 & 2.23 & 1.96 & --- & --- & --- & --- & --- & --- & --- & --- & --- \\
\hline T4 & 8.41 & 7.80 & 6.63 & 6.16 & 3.29 & 2.79 & 2.66 & 2.41 & 2.38 & 2.31 & --- & --- & --- & --- & --- & --- & --- & --- & -- \\
\hline T5 & 9.31 & 8.31 & 7.11 & 6.75 & 4.74 & 3.60 & 2.70 & 2.61 & 2.51 & 2.25 & --- & --- & --- & --- & --- & --- & --- & --- & --- \\
\hline T6 & 9.10 & 8.69 & 8.30 & 8.06 & 7.58 & 6.79 & 6.69 & 6.39 & 6.34 & 5.74 & 5.63 & 5.20 & 4.64 & 2.80 & 2.66 & 2.58 & 2.04 & 2.04 & 1.68 \\
\hline T7 & 9.50 & 9.19 & 8.98 & 8.73 & 8.50 & 8.11 & 7.69 & 7.08 & 6.38 & 5.84 & 5.60 & 5.00 & 4.70 & 4.38 & 2.88 & 2.70 & 2.39 & 2.13 & 1.41 \\
\hline T8 & 9.88 & 9.38 & 9.11 & 8.85 & 8.73 & 8.19 & 7.86 & 7.44 & 7.11 & 6.06 & 5.69 & 5.30 & 4.70 & 2.81 & 2.59 & 2.38 & 2.23 & 2.08 & 1.87 \\
\hline T9 & 10.75 & 10.38 & 10.01 & 9.58 & 8.78 & 8.09 & 7.86 & 7.21 & 6.85 & 6.71 & 6.10 & 5.99 & 4.31 & 2.74 & 2.50 & 2.39 & 2.45 & 1.94 & 1.91 \\
\hline S Em \pm & 0.49 & 0.30 & 0.29 & 0.26 & 0.24 & 0.22 & 0.19 & 0.16 & 0.14 & 0.10 & 0.10 & 0.10 & 0.07 & 0.05 & 0.06 & 0.07 & 0.07 & 0.09 & 0.06 \\
\hline CD $5 \%$ & 1.44 & 0.86 & 0.86 & 0.77 & 0.69 & 0.63 & 0.55 & 0.47 & 0.41 & 0.29 & 0.30 & 0.30 & 0.20 & 0.15 & 0.17 & 0.19 & 0.19 & 0.26 & 0.17 \\
\hline
\end{tabular}

Initial firmness: 11.12 
Table.5 Effect of 1-methylcyclopropene and polyhexamethylene guanidine on moisture (\%) content of banana fruits during storage

\begin{tabular}{|c|c|c|c|c|c|c|c|c|c|c|c|c|c|c|c|c|c|c|c|}
\hline Treatments & D2 & D3 & D4 & D5 & D6 & D7 & D8 & D9 & D10 & D11 & D12 & D13 & D14 & D15 & D16 & D17 & D18 & D19 & D20 \\
\hline T1 & 64.60 & 65.29 & 67.08 & 69.34 & 69.51 & 71.75 & --- & --- & --- & --- & --- & --- & --- & --- & --- & --- & --- & --- & --- \\
\hline T2 & 64.25 & 65.25 & 67.00 & 69.13 & 69.29 & 70.92 & 70.92 & 72.10 & 69.49 & 68.59 & --- & --- & --- & --- & --- & --- & --- & --- & --- \\
\hline T3 & 64.25 & 65.50 & 66.75 & 69.00 & 69.15 & 70.97 & 71.22 & 72.04 & 69.38 & 68.95 & --- & --- & --- & --- & --- & --- & --- & --- & --- \\
\hline T4 & 64.13 & 65.13 & 67.50 & 68.75 & 68.94 & 70.28 & 70.53 & 72.03 & 69.15 & 68.70 & --- & --- & --- & --- & --- & --- & --- & --- & --- \\
\hline T5 & 64.38 & 65.13 & 67.50 & 68.50 & 68.94 & 70.75 & 71.10 & 71.98 & 69.09 & 68.84 & --- & --- & --- & --- & --- & --- & --- & --- & --- \\
\hline T6 & 63.63 & 64.00 & 64.38 & 64.63 & 64.88 & 65.38 & 65.63 & 65.88 & 66.38 & 66.75 & 67.25 & 67.75 & 68.00 & 68.25 & 69.00 & 69.75 & 70.75 & 71.50 & 71.00 \\
\hline T7 & 63.50 & 64.25 & 64.88 & 65.13 & 65.38 & 65.63 & 65.88 & 66.13 & 66.38 & 66.63 & 66.88 & 67.25 & 67.50 & 67.75 & 68.75 & 69.00 & 69.50 & 70.00 & 69.50 \\
\hline T8 & 63.54 & 63.79 & 64.53 & 64.78 & 65.28 & 65.75 & 65.75 & 65.75 & 66.00 & 66.25 & 66.63 & 66.88 & 67.13 & 67.25 & 67.75 & 68.00 & 68.75 & 69.25 & 69.25 \\
\hline T9 & 63.78 & 64.28 & 64.31 & 64.53 & 64.78 & 65.03 & 65.25 & 65.50 & 65.75 & 66.00 & 66.25 & 66.50 & 66.75 & 66.88 & 67.00 & 67.25 & 67.75 & 69.00 & 68.75 \\
\hline S Em \pm & 0.45 & 0.60 & 0.70 & 0.60 & 0.66 & 0.55 & 0.50 & 0.32 & 0.23 & 0.35 & 0.20 & 0.28 & 0.21 & 0.19 & 0.02 & 0.01 & 0.01 & 0.01 & 0.64 \\
\hline CD 5\% & 1.20 & 1.80 & 2.04 & 1.75 & 1.94 & 1.60 & 1.47 & 0.94 & 0.68 & 1.00 & 0.59 & 0.83 & 0.62 & 0.54 & 0.05 & 0.02 & 0.02 & 0.02 & 1.87 \\
\hline
\end{tabular}

Initial moisture: $63 \%$

Table 6. Effect of 1-methylcyclopropene and polyhexamethylene guanidine on decay loss (\%) of banana fruits during storage

\begin{tabular}{|c|c|c|c|c|c|c|c|c|c|c|c|c|c|c|c|c|}
\hline Treatment & D5 & D6 & D7 & D8 & D9 & D10 & D11 & D12 & D13 & D14 & D15 & D16 & D17 & D18 & D19 & D20 \\
\hline T1 & 16.66 & 24.99 & 34.59 & --- & --- & --- & --- & --- & --- & --- & --- & --- & --- & --- & --- & --- \\
\hline $\mathbf{T} 2$ & 2.44 & 2.78 & 2.78 & 2.78 & 2.78 & 6.79 & 11.43 & --- & --- & --- & --- & --- & --- & --- & --- & --- \\
\hline T3 & 0.00 & 0.00 & 5.72 & 5.72 & 5.72 & 7.85 & 7.85 & --- & --- & --- & --- & --- & --- & --- & --- & --- \\
\hline $\mathbf{T 4}$ & 0.00 & 0.00 & 2.56 & 2.56 & 2.56 & 2.56 & 3.44 & --- & --- & --- & --- & --- & --- & --- & --- & --- \\
\hline T5 & 0.00 & 0.00 & 2.13 & 2.13 & 2.13 & 2.13 & 2.13 & --- & --- & --- & --- & --- & --- & --- & --- & --- \\
\hline T6 & 0.00 & 0.00 & 8.58 & 8.58 & 13.83 & 13.83 & 13.83 & 13.83 & 13.83 & 13.83 & 26.83 & 26.83 & 26.83 & 26.83 & 26.83 & 26.83 \\
\hline T7 & 3.34 & 3.34 & 3.34 & 3.34 & 3.34 & 8.58 & 8.58 & 14.93 & 14.93 & 14.93 & 18.90 & 18.90 & 18.90 & 18.90 & 18.90 & 18.90 \\
\hline T8 & 0.00 & 0.00 & 0.00 & 0.00 & 0.00 & 0.00 & 0.00 & 0.00 & 0.00 & 0.00 & 7.43 & 7.43 & 7.43 & 7.43 & 7.43 & 7.43 \\
\hline T9 & 3.34 & 3.34 & 3.34 & 3.34 & 3.34 & 10.08 & 10.08 & 12.78 & 12.78 & 16.63 & 29.54 & 29.54 & 29.54 & 30.24 & 30.24 & 30.24 \\
\hline S. Em \pm & 0.004 & 0.006 & 0.008 & 0.007 & 0.007 & 0.006 & 0.007 & 0.004 & 0.004 & 0.004 & 0.003 & 0.003 & 0.003 & 0.004 & 0.004 & 0.004 \\
\hline $\begin{array}{c}\text { CD@ } \\
5 \%\end{array}$ & 0.013 & 0.018 & 0.022 & 0.020 & 0.019 & 0.018 & 0.020 & 0.013 & 0.013 & 0.013 & 0.008 & 0.008 & 0.008 & 0.013 & 0.013 & 0.013 \\
\hline
\end{tabular}


Table.7 Effect of 1-methylcyclopropene and polyhexamethylene guanidine on shelf life of banana fruits during storage

\begin{tabular}{cc}
\hline Treatments & No. of days \\
\hline $\mathrm{T}_{1}$ Control & 7.00 \\
$\mathrm{~T}_{2}$ PHMG @ 0.5\% & 11.00 \\
$\mathrm{~T}_{3}$ PHMG @ 1.0\% & 11.00 \\
$\mathrm{~T}_{4}$ PHMG @ 1.5\% & 11.00 \\
$\mathrm{~T}_{5}$ PHMG @ 2.0\% & 11.00 \\
$\mathrm{~T}_{6} 1-\mathrm{MCP} @ 05 \mathrm{ppm}$ & 20.00 \\
$\mathrm{~T}_{7} 1-\mathrm{MCP} @ 10 \mathrm{ppm}$ & 20.00 \\
$\mathrm{~T}_{8} 1-\mathrm{MCP} @ 15 \mathrm{ppm}$ & 20.00 \\
$\mathrm{~T}_{9} 1-\mathrm{MCP} @ 20 \mathrm{ppm}$ & 20.00 \\
\hline S. Em \pm & 0.45 \\
CD @ 5\% & 1.31 \\
\hline
\end{tabular}

Table.8 Regression studies on interaction effect of 1-MCP and PHMG on postharvest parameters with shelf life of banana

\begin{tabular}{|c|c|c|c|c|c|c|c|c|c|c|c|c|c|c|c|c|c|c|}
\hline & 1 & 2 & 3 & 4 & 5 & 6 & 7 & 8 & 9 & 10 & 11 & 12 & 13 & 14 & 15 & 16 & 17 & $\overline{18}$ \\
\hline Treatment & 1 & & & & & & & & & & & & & & & & & \\
\hline $\begin{array}{l}\text { Ripening } \\
\text { rate }\end{array}$ & $0.21^{* *}$ & 1 & & & & & & & & & & & & & & & & \\
\hline Respiration & $-0.12^{*}$ & $0.55^{* *}$ & 1 & & & & & & & & & & & & & & & \\
\hline Colour & $0.16^{* *}$ & $0.68^{* *}$ & $0.70^{* *}$ & 1 & & & & & & & & & & & & & & \\
\hline PLW & $0.36^{* *}$ & $0.64^{* *}$ & $0.51^{* *}$ & $0.81^{* *}$ & 1 & & & & & & & & & & & & & \\
\hline Moisture & $0.48^{* *}$ & $0.57^{* *}$ & $0.38^{* *}$ & $0.72^{* *}$ & $0.60^{* * *}$ & 1 & & & & & & & & & & & & \\
\hline Firmness & $0.53^{* *}$ & $0.01^{\mathrm{NS}}$ & $-0.32^{* *}$ & $0.02^{\mathrm{NS}}$ & $0.02^{\mathrm{NS}}$ & $0.59^{* *}$ & 1 & & & & & & & & & & & \\
\hline Acidity & $0.33^{* *}$ & $0.69^{* *}$ & $0.60^{* *}$ & $0.84^{* *}$ & $0.79^{* *}$ & $0.85^{* *}$ & $0.18^{* *}$ & 1 & & & & & & & & & & \\
\hline $\begin{array}{l}\text { Ascorbic } \\
\text { Acid }\end{array}$ & $0.22^{* *}$ & $0.39^{* *}$ & $0.30^{* *}$ & $0.52^{* *}$ & $0.26^{* *}$ & $0.84^{* *}$ & $0.60^{* *}$ & $0.62^{* *}$ & 1 & & & & & & & & & \\
\hline TSS & $0.14^{*}$ & $0.64^{* *}$ & $0.69^{* *}$ & $0.81^{* *}$ & $0.69^{* *}$ & $0.64^{* *}$ & $-0.10^{\mathrm{NS}}$ & $0.83^{* *}$ & $0.44^{* *}$ & 1 & & & & & & & & \\
\hline $\begin{array}{l}\text { Reducing } \\
\text { Sugar }\end{array}$ & $-0.02^{\mathrm{NS}}$ & $0.65^{* *}$ & $0.78^{* *}$ & $0.83^{* *}$ & $0.69^{* *}$ & $0.47^{* *}$ & $-0.37^{* * *}$ & $0.77^{* *}$ & $0.32^{* *}$ & $0.84^{* *}$ & 1 & & & & & & & \\
\hline Total sugar & $0.02^{\mathrm{NS}}$ & $0.64^{* *}$ & $0.75^{* *}$ & $0.76^{* *}$ & $0.72^{* *}$ & $0.43^{* *}$ & $-0.42^{* *}$ & $0.78^{* *}$ & $0.18^{* *}$ & $0.83^{* *}$ & $0.93^{* *}$ & 1 & & & & & & \\
\hline Pectin & $0.14^{*}$ & $-0.05^{\mathrm{NS}}$ & $-0.22^{* *}$ & $-0.04^{\mathrm{NS}}$ & $-0.14^{*}$ & $0.26^{* *}$ & $0.49^{* *}$ & $-0.09^{\mathrm{NS}}$ & $0.42^{* *}$ & $-0.19^{* *}$ & $-0.29^{* *}$ & $-0.36^{* *}$ & 1 & & & & & \\
\hline $\mathbf{C a}$ & $0.15^{* *}$ & $-0.06^{\mathrm{NS}}$ & $-0.22^{* *}$ & $-0.05^{\mathrm{NS}}$ & $-0.13^{*}$ & $0.24^{* *}$ & $0.45^{* *}$ & $-0.04^{\mathrm{NS}}$ & $0.41^{* *}$ & $-0.18^{* *}$ & $-0.24^{* *}$ & $-0.34^{* *}$ & $0.89^{* *}$ & 1 & & & & \\
\hline Mg & $0.15^{* *}$ & $-0.03^{\mathrm{NS}}$ & $-0.16^{* *}$ & $-0.01^{\mathrm{NS}}$ & $-0.18^{*}$ & $0.26^{* *}$ & $0.44^{* *}$ & $-0.06^{\mathrm{NS}}$ & $0.45^{* *}$ & $-0.15^{* *}$ & $-0.24^{* *}$ & $-0.32^{* *}$ & $0.96^{* *}$ & $0.90^{* *}$ & 1 & & & \\
\hline $\mathbf{P}$ & $0.12^{*}$ & $-0.02^{\mathrm{NS}}$ & $-0.16^{* *}$ & $0.03^{\mathrm{NS}}$ & $-0.05^{\mathrm{NS}}$ & $0.24^{* *}$ & $0.41^{* *}$ & $-0.04^{\mathrm{NS}}$ & $0.43^{* *}$ & $-0.15^{* *}$ & $-0.22^{* *}$ & $-0.30^{* *}$ & $0.94^{* *}$ & $0.89^{* *}$ & $0.96^{* *}$ & 1 & & \\
\hline Decay loss & $-0.08^{\mathrm{NS}}$ & $0.53^{* *}$ & $0.42^{* *}$ & $0.70^{* *}$ & $0.71^{* *}$ & $0.37^{* *}$ & $-0.30^{\text {** }}$ & $0.67^{* *}$ & $0.17^{* *}$ & $0.67^{* *}$ & $0.79^{* *}$ & $0.77^{* *}$ & $-0.35^{* *}$ & $-0.31^{* *}$ & $-0.32^{* *}$ & $-0.26^{* *}$ & 1 & \\
\hline Shelf life & $0.19^{* *}$ & $0.43^{* *}$ & $0.42^{* *}$ & $0.56^{* *}$ & $0.85^{* *}$ & $0.16^{* *}$ & $-0.29^{* *}$ & $0.52^{* *}$ & $-0.15^{* *}$ & $0.49^{* *}$ & $0.58^{* *}$ & $0.62^{* *}$ & $0.43^{* *}$ & $0.35^{* *}$ & $0.39^{* *}$ & $0.29^{* *}$ & $0.64^{* *}$ & 1 \\
\hline
\end{tabular}


Fig.1 Banana fruits treated with 1-MCP+PHMG at 1, 5 and 15 days of storage
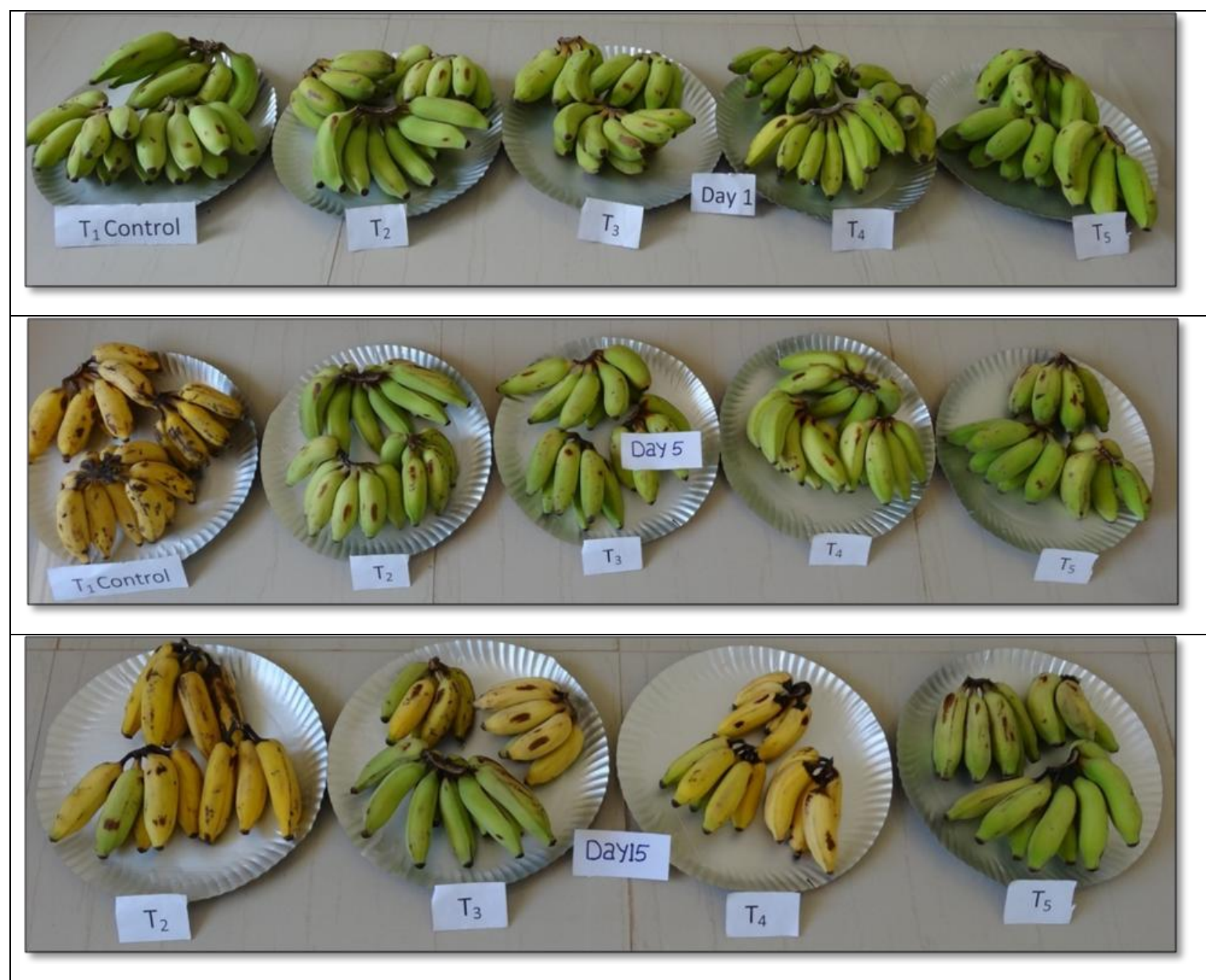
Effect of 1-MCP and PHMG on chlorophyll degradation

The chlorophyll content of banana fruit decreased with fruit ripening, the green pigment in unripe banana converted to carotenoids which is responsible for the characteristic yellow colour of ripe banana fruits.

Control fruits had shown decrease in chlorophyll content from day 1 to the $7^{\text {th }}$ day of storage while, chlorophyll content significantly higher inall the other treatment. Later it decreased on $15^{\text {th }}$ day of storage as shown in Fig.1.

Effect of 1-MCP and PHMG on physiological loss in weight, firmness and moisture Content

The physiological loss in weight (PLW) of 1MCP and PHMG treated fruits was significantly lower than the control fruits during storage Table 3 . The physiological loss in weight results mainly by the respiration and transpiration losses during the metabolic processes of fruits and also by atmospheric storage condition in terms of low relative humidity triggers the pressure difference between fruits and surrounding storage condition (Baile, 1975).

There was a significant decrease in firmness of banana fruits throughout the storage period as shown in the Fig. 2. The control fruits showed a decline in firmness from $11.7 \mathrm{~kg}$ per $\mathrm{cm}^{2}$ at first stage to $1.80 \mathrm{~kg}$ per $\mathrm{cm}^{2}$ ) on the $7^{\text {th }}$ day (Table 4). However, firmness was retained to a greater extent in 1-MCP and PHMG treatments. The firmness of the fruit tissue at harvest is mainly due to the physical properties of the individual cell walls and the middle lamella which contains the cementing pectic material (Sterling, 1975). As the fruit approaches ripening the tissue become soft due to the degradation of the cell wall and the intercellular adhesive substances (Pantastico, 1975; Sterling, 1975).

The moisture content of banana fruits increased during ripening as presented in Table 5, this increase in moisture content is expected, as water arising from the osmotic withdrawal of moisture from the peel is reported to exceed the net water lost in banana pulp due to transpiration (Loesecke, 1950). The moisture content of banana fruit recorded significant variation among treatment during banana storage. The moisture content of untreated fruits was recorded higher compared to the treated fruits on $7^{\text {th }}$ day of storage.

\section{Decay loss (\%)}

The highest decay loss was recorded in untreated fruits up to the $7^{\text {th }}$ day of storage and the fruits were discarded on $8^{\text {th }}$ day (Table 6). While combination effect of 1MCP and PHMG had shown significantly lower decay loss at the end of storage period. The 1-MCP and PHMG treated fruits had an extended storage life and lower spoilage intensity this is due to the delay in ripening caused by ethylene inhibition which might have increased its natural resistance to microbial infection.

\section{Effect of 1-MCP and PHMG on storage life}

Banana treated with 1-MCP and PHMG had shown a significant increase in storage life of 15 days at all treatments compared to control. The storage life (days to reach full ripe stage) of untreated fruits were found to be 7 days (Table 7). However, 1-MCP and PHMG treated fruits took 15 days to reach full ripe stage (Fig. 1). Shelf life of banana is interconnected with broader parameter such as physical, chemical, physiological and microbiological. In the present study it was 
observed that, the dependent variable was shelf life and it was related to many different independent parameters as shown in Table 8. Shelf life exhibited positive and significant association with ripening rate $(0.43)$, colour change (0.56), PLW (0.85), moisture (0.16), TSS (0.49), total sugars (0.62), pectin (0.43), calcium (0.35), potassium (0.29) and decay loss (0.64) while, it also showed negative and significant association with firmness $(-0.29)$, ascorbic acid (-0.15).

In conclusion the storage life of banana fruits can be extended up to 15 days if pre-treated with 1-MCPand decay loss can be minimized to a larger extent when treated with PHMG. The best treatment combination of 1-MCP and PHMG on banana fruits had shown an increased storage life and reduced microbial contamination till the end of the storage period. This treatment had reduced the fruit respiration rate, physiological weight loss and low decay loss and at the same time retained firmness and had higher concentrations of ascorbic acid, total soluble solids, total sugars, pectin and minerals however gave poor acceptability scores for colour, taste, texture, flavor and overall.

\section{Highlights}

1-methyl cyclo propene significantly delayed the onset of ripening rate, respiration rate and colour change and poly hexa methylene guanidine showed the best results in reducing pathological breakdown and extended the shelf life up to 15 days with acceptable eating quality.

\section{References}

AOAC. 1980. Official methods of analysis. $15^{\text {th }}$ edn. Association of Official Analytical Chemists (AOAC); Washington DC.

Baile, J.B. 1975. Synthetic and degradation process in fruit ripening. In Postharvest biology and handling of fruits and vegetables. Avi. Pub. Co., Westport Connecticut USA.

Barrett, D.M., Beaulieu, J.C. and Shewfelt, R. 2010. Color, flavor, texture and nutritional quality of fresh-cut fruits and vegetables: Desirable levels, instrumental and sensory measurement and the effects of processing. Critical Reviews in Food Sci. Nutri., 50: 369389.

Barry, C.S., Giovannoni, J.J. 2007. Ethylene and fruit ripening. J. Plant Growth Regulation, 26: 143-159.

Chun, T.W. 2010. An overview of post harvest biology and technology of fruits and vegetables, Workshop on technology of reducing post harvest losses and maintaining quality of fruits and vegetables. 2-11.

Clara, P., Eduardo, V., Vilas, B., Mohamed B., Kader, A. 2002. Variability in responses of partially ripe bananas to 1 methylcyclopropene. Postharvest Biol. Technol. 28:75-/85.

Eduardo, V., Boas, B. B., Kader, A. A. 2006. Effect of atmospheric modification, 1MCP and chemicals on quality of freshcut banana. Postharvest Biol. Technol. 39:155-162.

Kader, A.A., Saltveit, M.E. 2003. Respiration and exchange. In: Bartz, J. A., Brecht, J. K. (Ed.). Postharvest physiology and pathology of vegetables ( $2^{\text {nd }}$ ed. $)$. Marcel Dekker, Inc., New York, USA pp 7-23.

Kays, S.J., Paull, R.E. 2004. Postharvest Biol., Exon Press, USA pp 568.

Lin, Z., Zhong, S., Grierson, D. 2009. Recent advances in ethylene research. J. Exp. Botany, 60: 3311-3336.

Loesecke, H.V. 1950. In: Bananas. $2^{\text {nd }}$ Edn. Inter Sci., New York.

Mcmurchie, E.J., Mcglasson, W.B., Eaks, I.L. 1972. Treatment of fruit with propylene 
gives information about the biogenesis of ethylene. Nature, 237: 235-236.

Nelson, S.C. 2008. Post harvest rots of banana. Plant Dis., PD-54: pp 1-4.

Pantastico, E.B. 1975. Postharvest Physiology, Handling and Utilization of Tropical and Subtropical Fruits and Vegetables.The AVI publishing Co., West port, connection, USA, pp. 86-90.

Pereira Silva, D. F., Chamhum Salomão, L. C, De Siqueira, D. L., Cecon, P. R., Rocha, A. 2009. Potassium permanganate effects in postharvest conservation of the papaya cultivar Sunrise Golden Pesq. Agropec. Bras. Brasília, 44(7): 669-675.

Saltveit, M.E. 1999. Effect of ethylene on quality of fresh fruits and vegetables. Postharvest Biol. Technol. 15: 279-292.

Sisler, E.C., Serek, M. 1997. Inhibition of ethylene responses in plants at the receptor level: recent developments. Physiol. Pl. 100:577-582.

Sterling, C. 1975. Anatomy of toughness in plant tissue in postharvest biology and handling of fruits and vegetables.AVI pub. Co. Westport Connecticut. USA.

Sunder Raj, N., Nagaraju, S., Venkataram, M. N., Jaganath, M. K. 1972. Design and analysis of field experiment, University of Agri. Sci., Bangalore, pp. 141-167.

Usha Rani, M. and Kishor Kumar, M. 2013. Geographical indications in fruit crops A review. PARIPEX - Indian J. Res., (5): 148-149.

Wills, R.B., Lim, J.S., Greenfield, H. 1984. Changes in chemical composition of 'Cavendish' banana (Musa acuminate) during ripening. J. Food Biochem., 8: 69-77.

Wu, C.T. 1010. An overview of post harvest biology of fruits and vegetables. AARDO workshop on reducing post harvest losses and maintaining quality of fruits and vegetables. pp 2-11.

Yueming, J., Daryl, C. J., Andrew, J. M. 1999. Extension of the shelf life of banana fruit by 1- MCP in combination with polyethylene bags. Postharvest Biol. \& Technol., 16: 187-193.

\section{How to cite this article:}

Gouri, S.B., H.C. Krishna, T.H. Shankarappa, K.N. Sreenivas, G.K. Sadananda, D.V. Sudhakar Rao and Shamina Azeez. 2017. Effect of 1-Methylcyclopropene and Polyhexamethylene Guanidine on Postharvest Quality of Banana (Musa paradisiaca) cv. Yelakki. Int.J.Curr.Microbiol.App.Sci. 6(3): 1950-1961. doi: https://doi.org/10.20546/ijcmas.2017.603.222 